5
$\vdots$
$\vdots$
$\infty$

SSCL-641

September 1993

Distribution Category: 414

Yu. Senichev

Transient Effect in

High Intensity

Proton Linear Accelerators

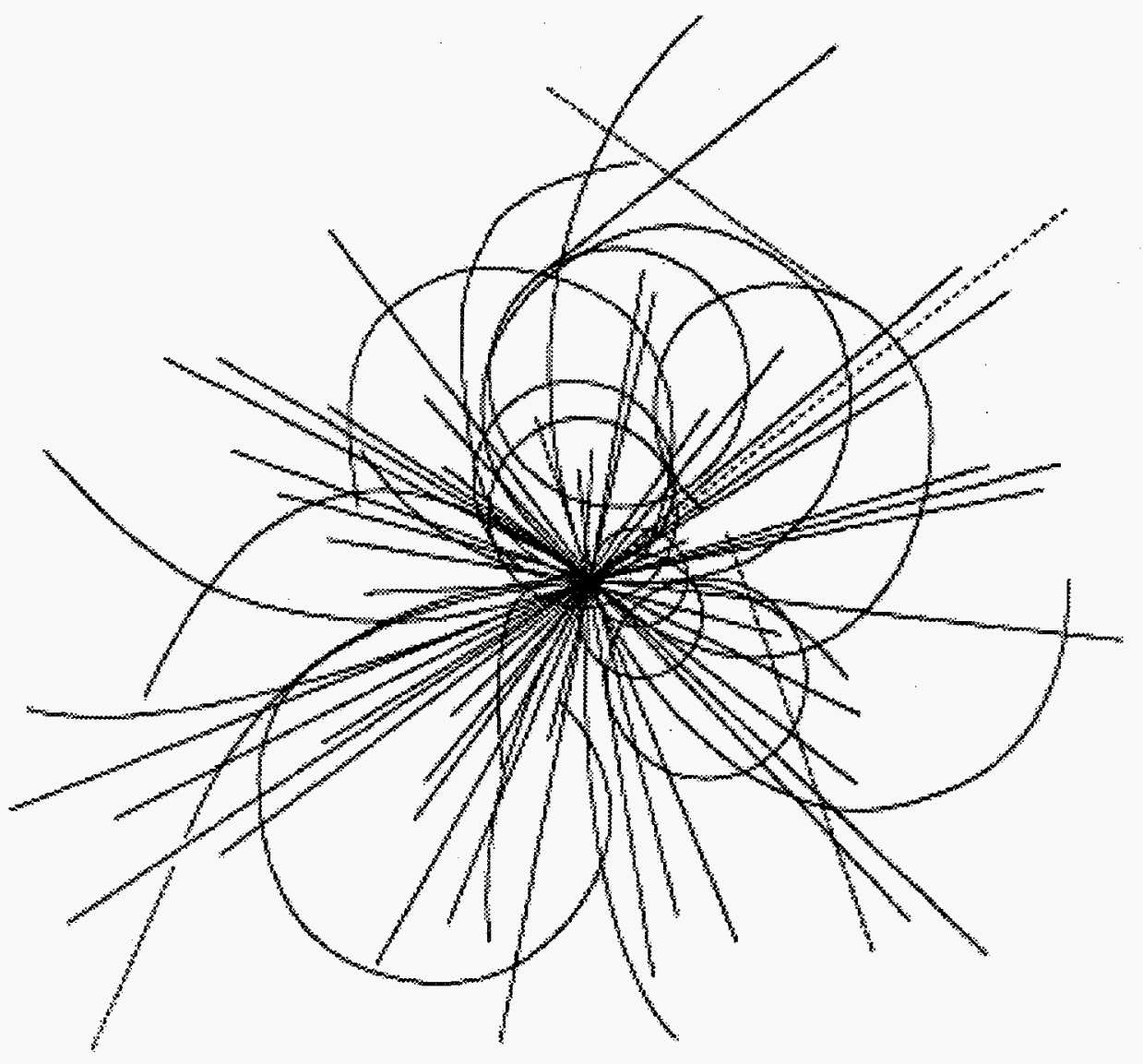

Superconducting Super Collider Laboratory 


\section{Disclaimer Notice}

This report was prepared as an account of work sponsored by an agency of the United States Govemment. Neither the United States Govemment or any agency thereot, nor any of their employees, makes any warranty, express or implied, or assumes any legal liability or responsibility for the accuracy, completeness, or usefulness of any information, apparatus, product, or process disclosed, or represents that its use would not infringe privately owned rights. Feference herein to any specific commercial product, process, or service by trade name, trademark, manufacturer, or otherwise, does not necessarily constitute or imply its endorsement, recommendation, or favoring by the United States Government of any agency thereof. The views and opinions of authors expressed herein do not necessarily state or reflect those of the United States Govemment or any agency thereof.

Superconducting Super Collider Laboratory is an equal opportunity employer. 


\title{
Transient Effect in High Intensity Proton Linear Accelerators*
}

\author{
Yu. Senichev \\ Superconducting Super Collider Laboratory ${ }^{\dagger}$ \\ 2550 Beckleymeade Avenue \\ Dallas, Texas 75237
}

September 1993

\begin{abstract}
DISCLAIMER
This report was prepared as an account of work sponsored by an agency of the United States Government. Neither the United States Government nor any agency thereof, nor any of their employees, makes any warranty, express or implied, or assumes any legal liability or responsibility for the accuracy, completeness, or usefulness of any information, apparatus, product, or process disclosed, or represents that its use would not infringe privately owned rights. Reference herein to any specific commercial product, process, or service by trade name, trademark, manufacturer, or otherwise does not necessarily constitute or imply its endorsement, recommendation, or favoring by the United States Government or any agency thereof. The views and opinions of authors expressed herein do not necessarily state or reflect those of the United States Government or any agency thereof.
\end{abstract}

* Also submitted to Particle Accelerators.

t Operated by the Universities Research Association, Inc., for the U.S. Department of Energy under Contract No. DE-AC35-89ER40486. The permanent address of Yu. Senichev is INR of Russian Academy of Science, Moscow. 


\section{DISCLAIMER}

Portions of this document may be illegible in electronic image products. Images are produced from the best available original document. 


\title{
Transient Effect in High Intensity Proton Linear Accelerators
}

\author{
Yu. Senichev
}

\begin{abstract}
We study the possible mechanism of the separatrix destruction during the transient in the linear accelerator. This effect is due to the parametric resonance of the beam in the longitudinal plane caused by the perturbations of electromagnetic field. The magnitude of time-space perturbations of the electromagnetic field depends on the disperse feature of resonator and the beam intensity. In the paper we discuss how to avoid this effect or to decrease its influence.
\end{abstract}




\subsection{INTRODUCTION}

Presently, the research of phenomena associated with high intensity proton linear accelerators requires new emphasis since many questions now need answers. One issue is the emittance growth due to the resonant features of the beam. ${ }^{1}$ The present proton linear accelerator development has made it possible to accelerate a beam current of some tens $\mathrm{mA}$ in pulse. Such effects as the beam pulse break due to excitation of the HEM wave and the beam loading on the fundamental mode now are design considerations.

The new tendencies are in terms of the accelerating rate increasing and using a more powerful generator to drive more long resonators. These considerations help increase accelerator efficiency. Since the efficiency is defined by the ratio of the beam power and the losses power, it is reasonable to increase this ratio. But on the other hand, when the beam loading is considered more strongly, feedback system complications result. So the next step is to raise the generator efficiency and power, and decrease the number of cavities, which automatically reduces the number of control systems as well. But a higher accelerating rate and a longer cavity results in a new effect-conservation violation of stable longitudinal motion which in turn can result in emittance growth. What is the substance of this effect?

For compensation of the time-dependent perturbation of the electromagnetic field during transients, the generator varies the power. However, this power has to excite all eigen waveguide modes, and spreads in both directions from the power input point which causes space-time-dependent perturbations. The magnitude of space perturbations of the electromagnetic field depends on the disperse features of the resonator and has the scale of the cavity length. At the same time, the longitudinal oscillation frequency depends on the electromagnetic field amplitude and grows with the rate acceleration. So for accelerators which have been constructed before with short resonators and low accelerating rates, the wavelength of perturbation is much less than the longitudinal oscillation wavelength. Increasing the accelerating rate and cavity length significantly increases the probability of parametric resonances in the longitudinal motion. These resonances even at a relatively small amplitude of perturbation $\sim 2 \div 5 \%$ can destroy the separatrix. When increasing the accelerating rate and decreasing resonator length, the order of synchrobetatron resonance falls, so the longitudinal oscillations can influence transverse motion and emittance growth.

This paper considers the instability of the beam due to parametric resonances in the longitudinal plane caused by the input of power for beam loading compensation. Transients can be caused not only by the beam switching on or off but also by the feedback system that has a high coefficient of stabilization. The suppressing of any amplitude or phase random instability by the feedback system leads to the input of corrected power, which 
causes the space distortion of the electromagnetic field. ${ }^{2}$ Concerning the beam instability, it is reasonable to start with the mechanism of instability. To emphasize the actuality of the problem and avoid abstraction, we begin with the reason for instability.

\subsection{ELECTROMAGNETIC FIELD PERTURBATION IN RESONATOR}

High energy proton linear accelerators mainly have two types of accelerating structures: Disk and Washer (DW) and Side Coupled (SC) cells. Both have many common features. So we consider this phenomenon in these structures. The perturbation of the electromagnetic field in the resonator can be described by a series of normal modes for the periodical chain of coupled cells (accelerating and coupling). ${ }^{3}$ Every cell is considered in a single mode approximation. So the field is represented as:

$$
\begin{aligned}
& E(x, y, z, t)=R e\left[\sum_{s}^{N} A_{s}(t) E_{0}(x, y, z) e^{i h_{s} z} e^{-i \omega t}\right], \\
& H(x, y, z, t)=\operatorname{Re}\left[\sum_{s}^{N} B_{s}(t) H_{0}(x, y, z) e^{i h_{s} z} e^{-i \omega t}\right],
\end{aligned}
$$

where $A_{s}(t), B_{s}(t)$ are electric and magnetic components time-dependent amplitudes of $s$-th mode which have distribution along cavity $E_{s}=E_{0}(x, y, z) e^{i h_{s} z}, H_{s}=H_{0}(x, y, z) e^{i h_{s} z}$, $w$ is the frequency of the outside source (beam or generator), $E_{0}(x, y, z)$ and $H_{0}(x, y, z)$ describe the field distribution in one cell, and $h_{s}$ is the wave number of $s$-th mode. The eigen functions $E_{s}$ and $H_{s}$ are connected with each other by Maxwell's homogeneous equations:

$$
\begin{aligned}
& \operatorname{curl} E_{s}=j \mu_{0} w_{s} H_{s}, \\
& \operatorname{curl} H_{s}=-j \varepsilon_{0} w_{s} E_{s},
\end{aligned}
$$

where $w_{s}$ is the eigen frequency of $s$-th mode. We primarily are interested in the electromagnetic field perturbation initiated by the beam switching on or off, although the following generalization is for the common case. We solve the problem of beam loading and compensation of the beam electromagnetic field perturbation by the generator. It is assumed that at the moment $t=0$, the beam enters the cavity. Simultaneously, the generator gives the additional power required to compensate for beam loading. In the common form, the total electromagnetic field is described by Maxwell's unhomogeneous equations. Substituting Eqs. (1)-(4) in Maxwell's unhomogeneous equations we get the expression for time-dependent coefficients $A_{s}(t)$ and $B_{s}(t)$ :

$$
\frac{\partial A_{s}}{\partial t}-j w A_{s}+j w_{s} B_{s}=-\frac{1}{N_{s}}\left[\int_{V} j^{g} E_{s}^{*} d v-\int_{V} j^{e} E_{s}^{*} d v\right],
$$




$$
\begin{gathered}
\frac{\partial B_{s}}{\partial t}-j w B_{s}+j w_{s} A_{s}=0 \\
N_{s}=\varepsilon \int_{V} E_{s} E_{s}^{*} d v
\end{gathered}
$$

where $j^{g}$ and $j^{e}$ are the generator and beam currents, and $N_{s}$ is a norm.

Eliminating $A_{s}$ and $B_{s}$ and taking into account the quality factor $Q_{s}$ in $w_{s}=w_{0 s}(1-$ $j / Q_{s}$ ) (below we omit index " 0 " in $w_{0 s}$ ) we can receive the following equations:

$$
\frac{\partial A_{s}}{\partial t}+\frac{j\left(w_{s}^{2}-w^{2}\right)+w_{s}^{2} / Q_{s}}{2 w} A_{s}=-\frac{1}{2 N_{s}} \int_{V} j^{g} E_{s}^{*} d v+\frac{w_{s}}{2 N_{s} w} \int_{V} j^{e} E_{s}^{*} d v
$$

Symmetrically one can get the expression for $B_{s}(t)$, but for the beam dynamic equation we need only to know $A_{s}(t)$. Solving this differential equation, we have:

$$
A_{s}(t)=\frac{Q_{s}}{N_{s} \omega_{s}} a_{s}(t)\left[\int_{V} j^{g} E_{s}^{*} d v+\int_{V} j^{e} E_{s}^{*} d v\right]
$$

where

$$
\begin{gathered}
a_{s}(t)=\frac{1-e^{-i \frac{\omega_{s}^{2}-\omega^{2}}{2 \omega} t} e^{-\frac{\omega_{s}^{2}}{2 \omega Q_{s}} t}}{1+i \frac{\omega_{s}^{2}-\omega^{2}}{\omega_{s}^{2}} Q_{s}}, \\
\omega_{s}^{2}-\omega^{2}=K_{f} \cos \left(h_{s}\right),
\end{gathered}
$$

where $K_{f}$ is the coupling coefficient. The first integral describes the additional power of the generator and the second one is the electromagnetic field excitation by the beam. The coefficients outside the integrals are the same, so the beam loading compensation in the trivial case is when the integrals cancel. However, in practice it is possible to compensate only for one mode, since the character of the interaction of the beam and of the generator with the resonator is quite different. Actually, the beam and the generator currents can be written as

$$
\begin{aligned}
& j^{e}(z, t)=j_{1}(z) e^{-i\left(\omega t-h_{b} z\right)} \\
& j^{g}(z, t)=j_{1} \delta\left(z-z_{0}\right) e^{-i \omega t}
\end{aligned}
$$

where $h_{b}=\omega / v_{b}, v_{b}$ is the beam velocity, $z_{0}$ is the point of power input into the resonator, $j_{1}$ means the first harmonic, the frequency of the beam, and the generator all are equal to each other. Here the magnitude of the currents is taken as an average over the cross section of the beam and the loop (or hole), since we study the excitation of axial symmetrical modes only. It is obvious that the integral of the beam interaction for a constant first harmonic along the resonator doesn't equal zero except for the fundamental mode $\left(s=s_{f}\right)$ under the condition when $h_{s}=h_{v}$. At the same time, the integral for the generator doesn't equal 
zero for any mode if the coupling coefficient $\operatorname{Re}\left[e^{i h_{s} z_{0}}\right]$ has a "nonzero" magnitude. In other words, in order to compensate for the beam perturbation of the fundamental mode, we have to input power that equals the beam power, but simultaneously this local input of power also excites all modes. Let's rewrite the expressions of Eqs. (1) and (9)-(11) for the field induced by the beam taking into account that only the fundamental mode will be excited.

$$
E_{b 1}=R e\left[\left(1-e^{-\frac{\omega}{2 Q_{0}} t}\right) e^{i h_{b} z} E_{0}(x, y, z) e^{-i\left(\omega t+\phi_{s}\right)} R P\right],
$$

where $R P=\frac{P_{\text {beam }}}{P_{\text {los }} \cos \phi_{s}}, Q_{0}$ is the loaded quality factor of the resonator for the fundamental mode, $P_{\text {beam }}=\frac{1}{2} \cos \phi_{s} \int_{v} j_{1}^{e} E_{s}^{*} d v$, and $\phi_{s}$ is the synchronous phase of the beam. Here we have taken $P_{l o s}=\frac{N_{s} \omega}{2 Q_{0}}$. Now since we are interested in the effect of the interaction of the beam with the perturbed field by itself, let's represent the field of the generator in the view of the traveling harmonics $E_{1}=0.5 E_{0}$ with the phase velocity equal to the beam velocity:

$$
E(x, y, z, t)=\operatorname{Re}\left[\sum_{s}^{N} B_{s}(z, t) 2 E_{1} e^{-i\left(\omega t-h_{b} z+\phi_{s}\right)}\right] R P,
$$

where

$$
\begin{aligned}
& B_{s}(z, t)=e^{-i\left[\left(\omega_{s}-\omega\right) t-\left(h_{s}-h_{b}\right) z\right]} \\
& \times \begin{cases}\left(1-e^{-\frac{\omega}{2 Q_{0}} t}\right) & \text { if } s=s_{f} \\
-\frac{e^{-\frac{\omega_{s}^{2}}{2 \omega_{s}} t}}{1+i \frac{\omega_{s}^{2}-\omega^{2}}{\omega^{2}} Q_{s}} & \text { otherwise. }\end{cases}
\end{aligned}
$$

The symmetry of $s$-th and $-s$-th modes relative to the fundamental mode is taken. This expression shows that the space-time distortion (front) of the electromagnetic field with the carrier resonant frequency $w$ moves along the resonator with a velocity equal to average velocity for all modes:

$$
v_{\text {front }}=\overline{\left(\omega_{s}-\omega\right) /\left(h_{s}-h_{b}\right)} .
$$

Since

$$
\lim _{\omega_{s} \rightarrow \omega \rightarrow 0}\left(\omega_{s}-\omega_{b}\right) /\left(h_{s}-h_{b}\right)=v_{\text {group }},
$$

then it means that the front of perturbation that the beam "sees" moves with the group velocity. It is obvious that each mode has its own group velocity and moreover, each mode damps slightly differently. Thus, the front will change shape as it moves along the resonator. $B_{s}(z, t)$ for $s=s_{f}$ is the average of the electromagnetic field over the length of the resonator at any moment in time. It is exactly equal to the field induced by the beam when $P_{\text {beam }}=P_{\text {gener }}$. So if this term is subtracted from Eq. (1), then we shall get the field that the beam sees. Knowing the dispersion function of the structure, it is possible 
to calculate what the distribution of the electromagnetic field will be during the transient. Figure 1 shows the field of the generator at the moment $t=0.4 \mu \mathrm{s}$. This illustrates what will happen in the cavity during injection of the beam and the simultaneous input of power. The origin of coordinates corresponds to the point of the power input. In the case of the Superconducting Super Collider (SSC), this is the middle of the resonator. Physically, it means that the beam moves with the phase velocity and fills the resonator $1 / K_{f}$ as fast as the wavefront from the generator. So after the first tens of ns the beam interacts with the whole resonator and the average level of the field will decrease in accordance with the exponential law $\sim\left(1-e^{-\omega t / 2 Q_{0}}\right)$. The generator creates a wave that radiates in both directions from the middle and divides the resonator into three parts with alternative drops of field. The overall change in time goes as $\sim e^{-\omega t / 2 Q_{0}}$. The main contribution to the distortion of the field in the resonator is caused by harmonics which are reflected with coefficients $\mid \rho /=1$. The incident and reflected waves give a standing wave:

$$
e^{i h_{s} z}+e^{-h_{s} z}=2 \cos \left(h_{s} z\right),
$$

which means that the distribution is described by $\cos \frac{\pi s}{N} n$, where $N$ is the total number of cells in a module and $n$ is the number of the cell. Figures 2 and 3 show the typical dispersion curve of a side coupled structure and distribution of electromagnetic field amplitude in accelerating cells for some modes. Figure 4 shows the absolute value of electromagnetic field distribution of first (a) and second (b) modes experimentally measured on low level of the power for the second tanks of the SSC Linac second module (the measurements have been done by Chu Rui Chang). The character is absolutely identical to the theoretically taken one. Besides, one can say that the integral over module of the total distortion (except the fundamental mode) equals zero. However, the front of the wave can have significant impact. For the simplest case when we use linear dispersion, $\omega_{s}-\omega=\frac{\pi}{2} \frac{\omega K_{f}}{N}$, it is possible to get an analytical expression for the height of the wave: ${ }^{3}$

$$
\delta E_{1}=2 E_{1} R P \frac{L_{\text {cav }}}{v_{\text {group }}} \frac{\omega}{2 Q_{0}} e^{-\omega t / 2 Q_{0}},
$$

or using $P_{\text {beam }}=\frac{1}{2} I_{1} E_{1} \cos \phi_{s}$, one gets the expression by taking $R_{\text {shunt }}=E_{1}^{2} L_{\text {cavity }} / P_{\text {losses }}$ :

$$
\delta E_{1}=I_{1} R_{\text {shunt }} \frac{\tau_{g r}}{\tau_{t}} e^{-t / \tau_{t}},
$$

where $\tau_{g r}=L_{c a v} / v_{g r}$ and $\tau_{t}=2 Q_{0} / \omega$. The value $K_{\tau}=\frac{\tau_{g r}}{\tau_{t}}$ defines the ratio between the front of the wave at the initial time and the final magnitude of the electromagnetic field obtained when we excite the resonator by a generator with the vector $I_{1} R_{\text {shunt }}$-for the SSC case $K_{\tau}=0.35$. This means inputting a generator power into the resonator 
with a shunt impedance $R_{\text {shunt }}=50 \mathrm{M} \Omega$ to compensate the beam loading with first a harmonic $I_{1}=2 I_{0}=2 * 0.02 \mathrm{~mA}$, then a wave is excited with a front amplitude that equals $0.7 \mathrm{MV} / \mathrm{m}$. This is $\sim 10 \%$ of the accelerating harmonic. In the next section we shall need the Fourier representation of perturbation. Using the common expression for perturbation, Eqs. (1) and (9)-(11), we can rewrite it as:

$$
\delta E_{1}(z, t)=E_{1} R P e^{-t / \tau} \sum_{s=1}^{N / 2} e_{k} \cos \frac{2 \pi k z}{L_{r}},
$$

$L_{r}$ is the resonator length and $e_{k} \simeq \frac{\operatorname{Sin} \Delta_{k} w t}{\Delta_{k} w / w_{s} Q_{s}}$, where $\Delta_{k} w=\left(w-w_{1}\right) k$, which coincides with one of $w-w_{s}$.

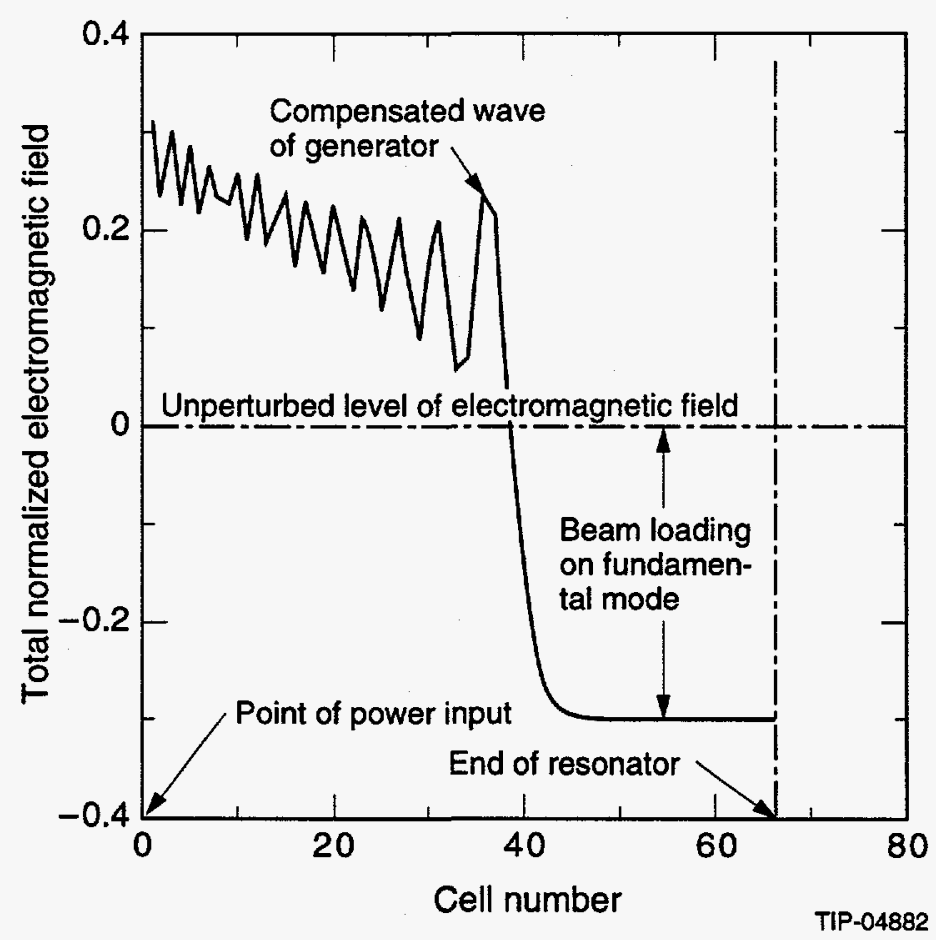

Figure 1. The perturbed electromagnetic field in resonator. 


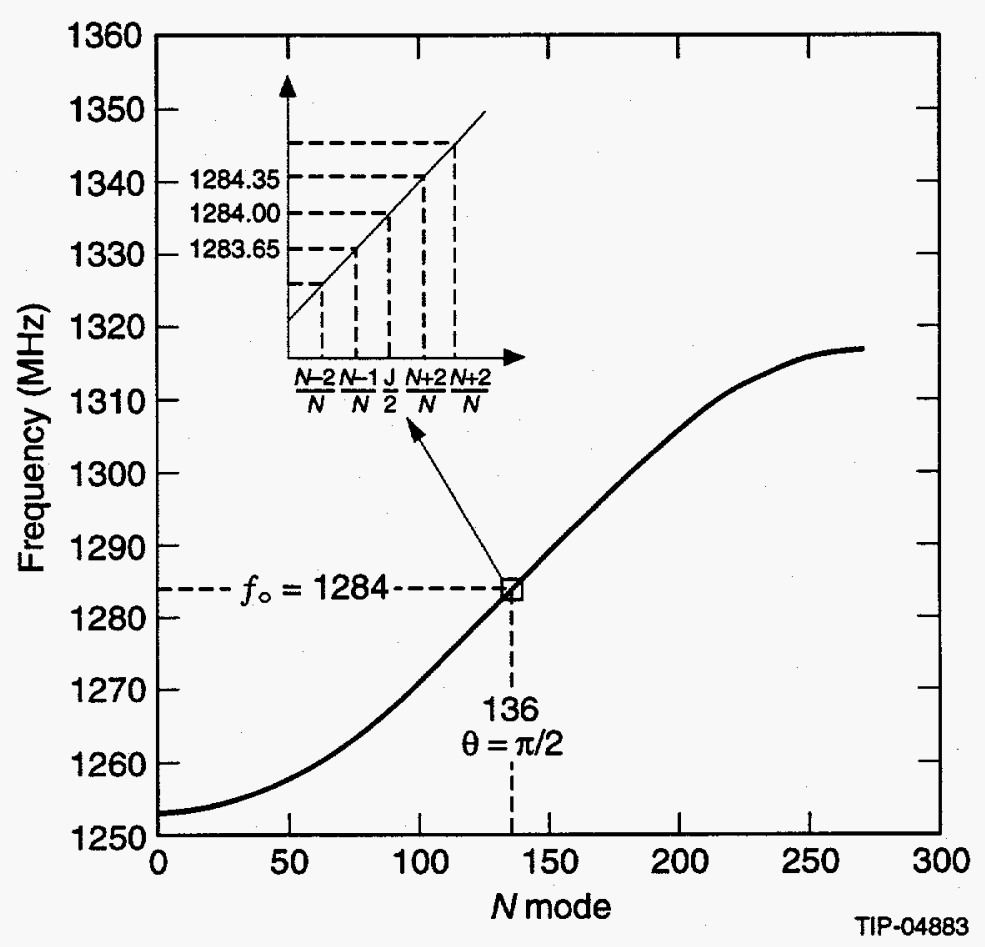

Figure 2. The dispersion curve of the side-coupled structure:

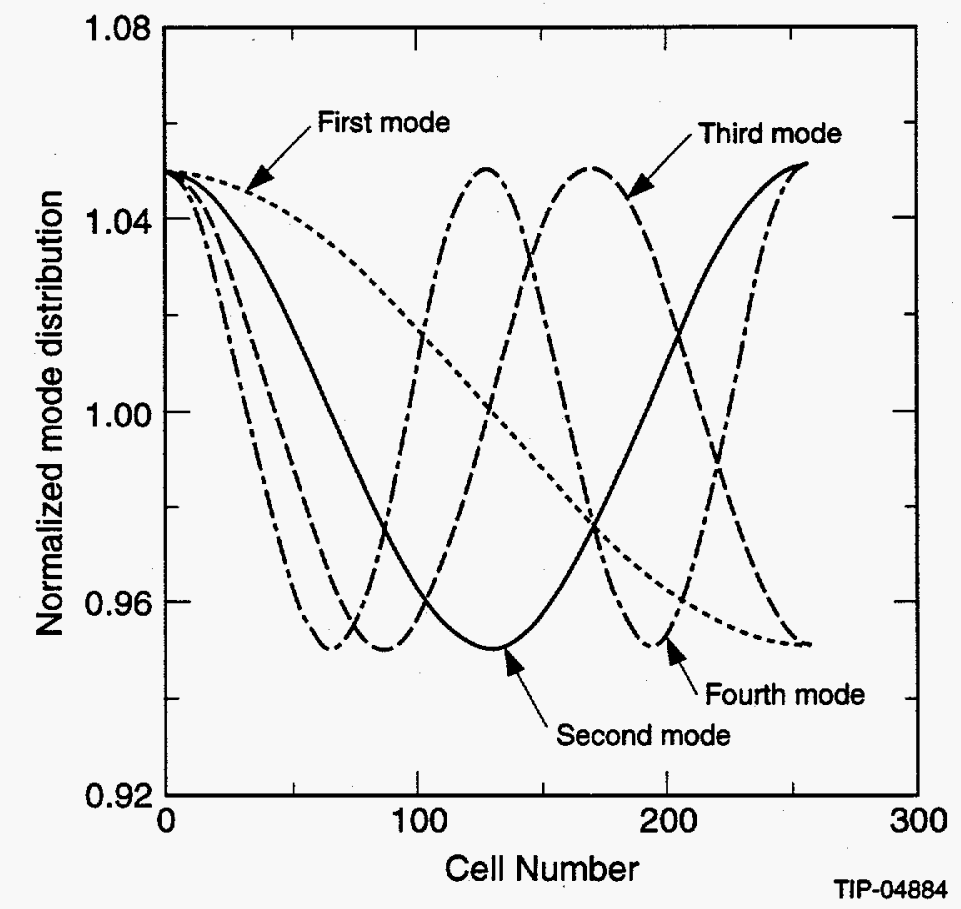

Figure 3. The distribution of electromagnetic field for 1-4 modes. 

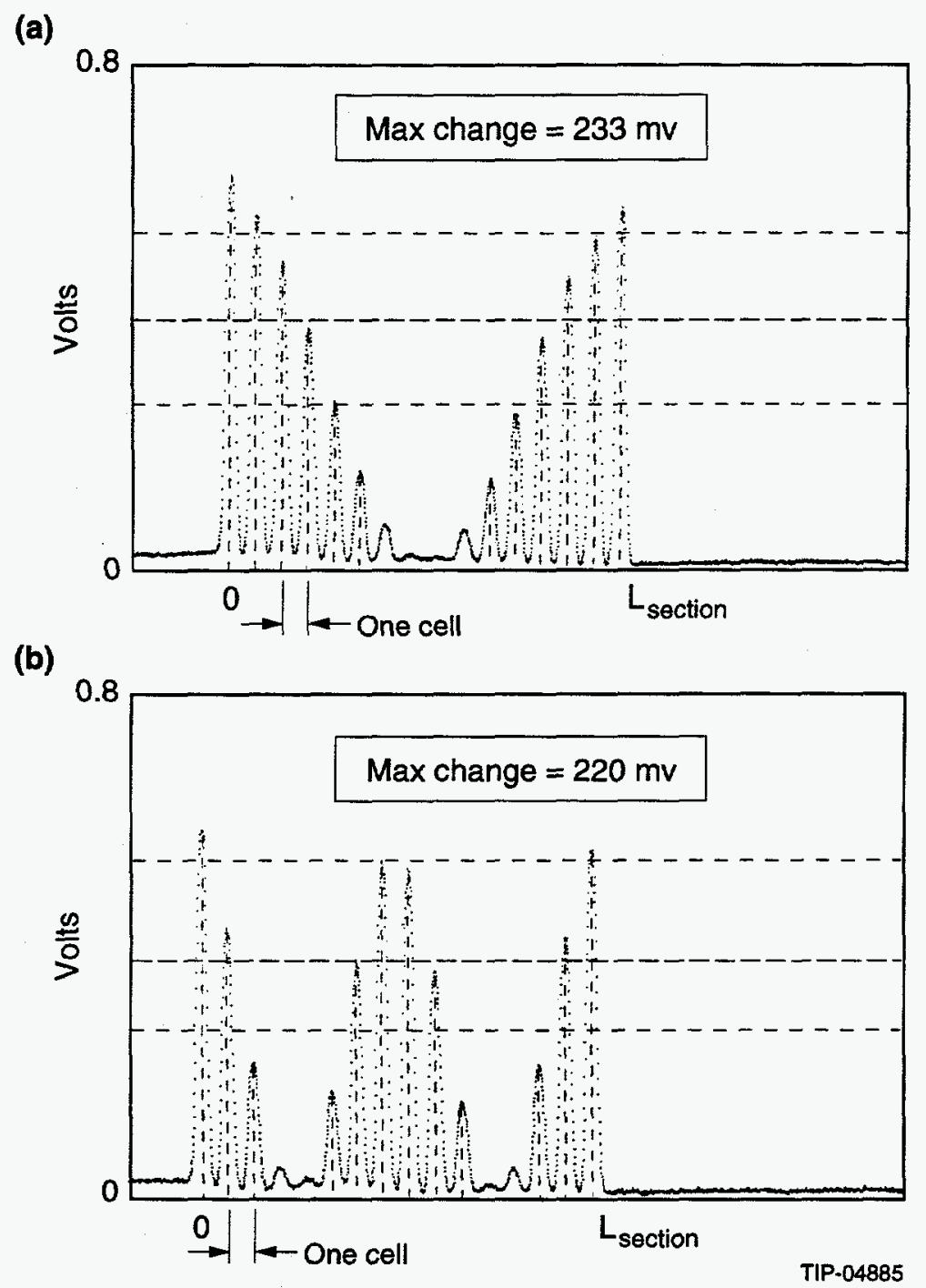

Figure 4. The experimental measurement of: (a) first mode electromagnetic field distribution and (b) second mode electromagnetic field distribution.

\subsection{PARAMETRIC RESONANCE AND LONGITUDINAL MOTION INSTABILITY}

\subsection{In Structure with Constant Phase Velocity}

Thus, during the transient the beam sees practically the rectangular wave that radiates from the point of power input and damps simultaneously (see Figure 1). In this section we shall analyze the beam dynamics in this perturbed field.

The DW or SC structures have many features. One of them is a constant cell size in each tank which implies a constant phase velocity along the tank. For simplification let's assume the distribution of the electromagnetic field in each accelerating cell doesn't depend 
on transverse coordinates. Although for the synchrobetatron resonance the inverse couple is important. It is presented as:

$$
E(z, t)=E(z) \sin w t(z)
$$

where

$$
t(z)=\frac{1}{c} \int_{0}^{z} \frac{d \xi}{\beta_{\text {part }}(\xi)}
$$

$t(z)$ is the current time in the coordinate $z$ for the particle, which moves with velocity $\beta_{\text {part }}$ in accordance with equation:

$$
\frac{d p(z, t)}{d t}=e E(z, t)
$$

$p=m_{0} \gamma \beta_{\text {part }} c$ is a momentum of particle. Let's represent the distribution of the electromagnetic field in the accelerating cell by the Fourier series:

$$
E(z)=\sum_{m=1}^{\infty} A_{m} \cos \frac{2 \pi m}{L} z
$$

where $L$ is the period length (for SC and DW, $L$ is double the length of the accelerating cell). Then substituting Eq. (26) in (23) we get:

$$
\begin{aligned}
E(z, t) & =\sum_{m=1}^{\infty} A_{m} \cos \frac{2 \pi m}{L} z \sin w t(z) \\
& =\frac{1}{2} \sum_{m=-\infty}^{\infty} A_{m} \sin \left[w t(z)-\frac{2 \pi m}{L} z\right],
\end{aligned}
$$

here $\frac{2 \pi m}{L} z$ can be considered as the wave number and is written as $k_{m}=\frac{w}{v_{m}}$, where $v_{m}$ is the phase velocity of the $m$-th harmonic. Then the motion equation is represented as:

$$
\frac{d p}{d t}=\frac{e}{2} \sum_{m} A_{m} \sin \left[w t(z)-k_{m} z\right]
$$

If the particle moves in synchronism with one of the harmonics we can use the method "slow phase" $-\phi$ (in Russian literature this method is called "method of averaging over phase"):

$$
\phi=\frac{w}{c}\left[\int_{0}^{z} \frac{d \xi}{\beta_{w}}-\int_{0}^{z} \frac{d \xi}{\beta_{p a r t}}\right]
$$

where $c \beta_{w}=\frac{w}{k_{m}}$ is the phase velocity of $m$-th resonant harmonic. Below we shall down index "part" and understand $\beta$ as the relative velocity of the particle. Uniting the expression of Eq. (30) with Eq. (29) we get the following equation system:

$$
\begin{aligned}
& \frac{d p}{d t}=\frac{e A_{m}}{2} \sin \phi, \\
& \frac{d \phi}{d t}=w\left[1-\frac{\beta_{w}}{\beta}\right],
\end{aligned}
$$


where $0.5 A_{m}$ is amplitude of the $m$-th harmonic, which maintains the acceleration of particles. In the discussion that follows, the resonant harmonic, which is responsible for the fundamental resonance, will be noted as $E_{0} T$, where $T$ is time flight factor.

At first let's consider the case when $\beta_{w}$ is constant. Differentiating the second equation of the system, Eq. (31) and substituting $\frac{d \beta}{d t}$ from the first equation:

$$
\frac{d \beta}{d t}=\frac{e E_{0} T}{m_{0} c \gamma^{3}} \sin \phi
$$

in the second equation, we receive the equation:

$$
\frac{d^{2} \phi}{d \tau^{2}}+\frac{e E_{0} T \beta_{w} \lambda}{2 \pi m_{0} c^{2} \gamma^{3} \beta^{2}} \sin \phi=0
$$

where $\tau$ is a new more convenient variable $\tau=w t$, or $\tau=\pi n$, where $n$ is the number of the cell. This equation describes the oscillation of a particle in the resonator with constant phase velocity and it coincides with the equation of pendulum. It has the exact decision through elliptical integrals:

$$
\begin{aligned}
\phi(a, \psi) & =\frac{8 \sqrt{q}}{1+q} \cos \psi-\frac{8 q^{3 / 2}}{3\left(1+q^{3}\right)} \cos 3 \psi+\ldots, \\
\frac{\Omega}{\Omega_{0}} & =\frac{\pi}{2 \mathcal{K}} \\
r & =\sin \phi_{\max } / 2
\end{aligned}
$$

here $r$ is a module of $\mathcal{K}$, which is the elliptical integral of the first kind, $q=e^{-\pi \frac{\kappa^{\prime}}{\mathcal{K}}}$ and $\mathcal{K}^{\prime}(r)=\mathcal{K}\left(r^{\prime}\right)$, where $r^{\prime}=\sqrt{1-r^{2}}$ and $\Omega_{0}^{2}=\frac{e E_{0} T \lambda}{2 \pi m_{0} c^{2} \gamma^{3} \beta_{w}}, \Omega$ is the frequency, which depends on the amplitude of oscillation. Using these formulas, one can calculate the motion of one particle together with constant phase velocity and the oscillation frequency. Figure 5 shows how the average synchronous frequency depends on the amplitude. The central particles oscillate with the maximum frequency equal to $\Omega_{0}$, the boundary ones have zero frequency. Obviously any perturbation with diapason $0 \div \Omega_{0}$ will be in the first order resonance with some particle. Although in the common case for multi-order resonance this is always so for $l$ and $k$ when $l \Omega+k \nu=0$. We are interested in the motion in the perturbed electromagnetic field, which can give the resonant condition when instead of constant $E_{0}$, we shall have the variation of electromagnetic field along the resonator:

$$
E=E_{0}+\delta E_{1}(z, t)=E_{0}\left(1+\frac{\delta E}{E} \sum_{k} e_{k} \cos k \nu \tau\right),
$$


where $\nu=2 \pi / T_{r}$. Some harmonic with number $k$ can be a reason for the parametric resonance on the length of resonator $T_{r}=\pi N$, where $N$ is the number cells in the resonator. Then Eq. (33) has view:

$$
\frac{d^{2} \phi}{d \tau^{2}}+\Omega_{0}^{2}\left(1+e_{k} \cos k \nu \tau\right) \sin \phi=0 .
$$

The Hamiltonian of the unperturbed motion has the standard form, which is used in the literature: ${ }^{4,5}$

$$
H(p, \phi)=\frac{p^{2}}{2}+\Omega_{0}^{2} \cos \phi
$$

or in a more convenient form for solution by the perturbation method:

$$
H(p, \phi)=\frac{p^{2}}{2}+\frac{\Omega_{0}^{2} \phi^{2}}{2 !}-\frac{\Omega_{0}^{2} \phi^{4}}{4 !}+\ldots
$$

Taking the standard exchange of the variables on the action integral $I$ and phase $\phi=$ $\sqrt{2 I / \Omega_{0}} \cos \theta$, where $\theta=\Omega \tau$, we get:

$$
H(I, \theta)=\Omega_{0}\left[I-\frac{I^{2}}{3 !} \cos ^{4} \theta+\ldots\right] .
$$

For the perturbed motion, when $\Omega_{0}^{2}=\left(1+e_{k} \cos \tau\right)$, we have:

$$
H(I, \theta, \tau)=\Omega_{0}\left[I+e_{k} I \cos ^{2} \theta \cos \tau_{1}-\frac{I^{2}}{6} \cos ^{4} \theta+\ldots\right] .
$$

After averaging over phase:

$$
H(I, \theta, \tau)=\Omega_{0}\left[I+\frac{e_{k}}{4} I \cos \psi-\frac{I^{2}}{16}+\ldots\right],
$$

where $\psi=2 \theta-\tau$ is again a slow resonant phase. So for any $k \nu=2 \Omega$ we shall have the parametric resonance. As distinct from the linear resonance, the nonlinear term exists here and this term $\sim I^{2}$ plays the stabilizing role for the nonlinear parametric resonance. From last equation one can show that the maximum amplitude will be proportional to $e_{k}$ and restricted by final value $8 e_{k}$. However, we see from here that the threshold for $e_{k}$ is absent when the resonance starts. This means that any small perturbation has to cause the distortion of the separatrix in a case of constant phase velocity. This is true, but we are more interested the stepped phase velocity case. 


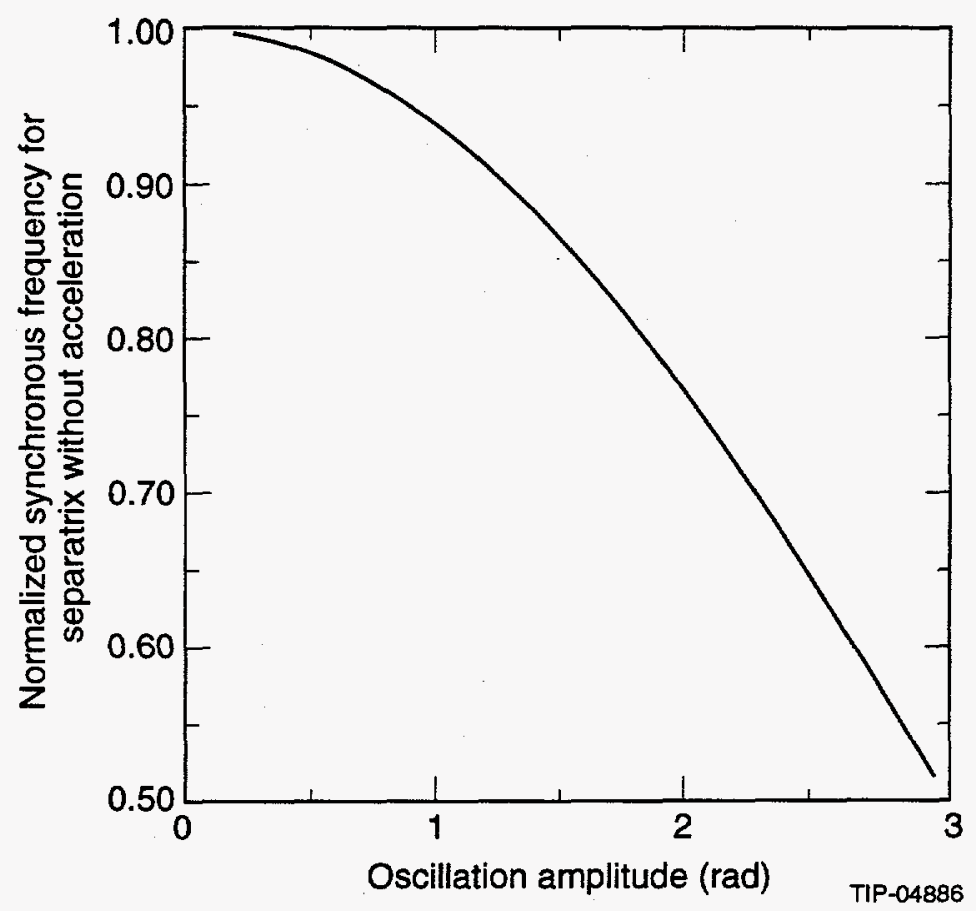

Figure 5. The average synchronous frequency vs. the oscillation amplitude.

\subsection{Stepped Phase Velocity Structure}

In reality, the phase velocity in structures such as DW and SC is changed step-bystep, remaining constant along each section. Figure 6 shows the periodical depending $\beta_{w}$ versus $\tau$. At the boundary of every section $\beta_{w}$ changes on $\delta \beta . T_{s}$ is a period equal to the section length. At the constant acceleration rate $\delta \beta$ practically doesn't depend on the beam energy $\left(\sim \frac{1}{\gamma^{3}}\right)$. Now let's return to the equation system (31), only now assuming a variation of the phase velocity $\beta_{w}$ and make the transformation in the reference system of "equivalent synchronous wave":

$$
\begin{aligned}
\frac{d^{2} \phi}{d \tau^{2}} & =-\frac{1}{\beta} \frac{d \beta_{w}}{d \tau}+\frac{\beta_{w}}{\beta^{2}} \frac{d \beta}{d \tau} \\
& =-\frac{1}{\beta} \frac{d \beta_{w}}{d \tau}+\frac{\beta_{w}}{\beta^{2}} \frac{d \beta}{d \tau}+\frac{\beta_{w}}{\beta^{2}} \frac{d \beta_{w}}{d \tau}-\frac{\beta_{w}}{\beta^{2}} \frac{d \beta_{w}}{d \tau} \\
& =-\frac{1}{\beta}\left(1-\frac{\beta_{w}}{\beta}\right) \frac{d \beta_{w}}{d \tau}+\frac{\beta_{w}}{\beta^{2}} \frac{d}{d \tau}\left(\beta-\beta_{w}\right) .
\end{aligned}
$$

Substituting the second equation of system (31) in this equation, we get:

$$
\frac{d^{2} \phi}{d \tau^{2}}+\frac{1}{\beta} \frac{d \beta_{w}}{d \tau} \frac{d \phi}{d \tau}+\frac{e E_{0} T \lambda \beta_{w}}{2 \pi m_{0} c^{2} \beta^{2} \gamma^{3}} \sin \phi-\frac{\beta_{w}}{\beta^{2}} \frac{d \beta_{w}}{d \tau}=0
$$


The phase velocity function can be represented as the sum of constantly growing function $\overline{\beta_{w}}$ and alternating component $\tilde{\beta_{w}}$ :

$$
\beta_{w}=\overline{\beta_{w}}+\tilde{\beta_{w}}=\alpha \tau+\sum_{n=1}^{\infty} \delta \beta \frac{1}{\pi n} \sin 2 \pi \nu_{\beta} n \tau
$$

where $\nu_{\beta}$ is the frequency of phase velocity changing. We used here the Fourier expansion of slow-wise $\beta_{w}$-function. Then:

$$
\frac{d \beta_{w}}{d \tau}=\alpha+2 \delta \beta \nu_{\beta} \sum_{n=1}^{\infty} \sin 2 \pi \nu_{\beta} n \tau .
$$

The particular solution of Eq. (43), taking into account Eq. (44) is:

$$
\alpha=\frac{e E_{0} T \lambda}{2 \pi m_{0} c^{2} \gamma^{3}} \sin \overline{\phi_{s}} \text {. }
$$

Thus finally we have the motion equation of a particle in an ideal accelerator with stepped phase velocity structure:

$$
\frac{d^{2} \phi}{d \tau^{2}}+\Omega_{0}^{2} \sin \overline{\phi_{s}} \frac{d \phi}{d \tau}+\Omega_{0}^{2}\left(\sin \phi-\sin \overline{\phi_{s}}\right)=2 \nu_{\beta} \delta \beta \sum_{n}^{\infty} \cos 2 \pi \nu_{\beta} n \tau
$$

From this equation we can see that all particles, even in the ideal structure without perturbation of the field, will do coherent oscillations. It is possible to show that the amplitude of this oscillation equals:

$$
\Delta \phi=\frac{\delta \beta}{\beta_{w}} \frac{1}{\Omega_{0}} \cot \frac{\Phi}{2} \frac{1-\cos \Phi / 2}{\cos \Phi / 2},
$$

$\Phi$ is advanced phase per $T_{s}$. In the case of a stepped phase velocity structure, when synchronous phase is not equal to zero, any perturbation of the electromagnetic field leads to not only a variation of synchronous frequency but to creation of external force as well. Actually, if we have the condition $E \sin \phi_{s}=$ const, then in the right side of the equation we shall have the additional term $\Omega_{0}^{2} \sin \overline{\phi_{s}} \frac{\delta E}{E}$. Then in the common case, when the electromagnetic field is perturbed, the equation of longitudinal motion has the form:

$$
\begin{aligned}
\frac{d^{2} \phi}{d \tau^{2}} & +\Omega_{0}^{2} \sin \overline{\phi_{s}} \frac{d \phi}{d \tau}+\Omega_{0}^{2}\left(1+\frac{\delta E}{E} \sum_{k} e_{k} \cos k \nu \tau\right)\left(\sin \phi-\sin \overline{\phi_{s}}\right) \\
& =2 \nu_{\beta} \delta \beta \sum_{n} \cos 2 \pi \nu_{\beta} n \tau+\frac{\delta E}{E} \Omega_{0}^{2} \sin \overline{\phi_{s}} \sum_{k} e_{k} \cos k \nu \tau .
\end{aligned}
$$

This is an unhomogeneous nonlinear equation with time-dependent coefficients. We shall solve this equation by an asymptotic method. ${ }^{6}$ For this one let's expand $\sin \phi$ around $\overline{\phi_{s}}$ :

$$
\sin \phi=\sin \overline{\phi_{s}}+\cos \overline{\phi_{s}} \psi-\frac{1}{2} \sin \overline{\phi_{s}} \psi^{2}-\frac{1}{3 !} \cos \overline{\phi_{s}} \psi^{3}+\ldots
$$


Retaining the square nonlinearity only and reducing into a linear view with the perturbation in the right side, we write:

$$
\frac{d^{2} \psi}{d \tau^{2}}+\Omega_{0}^{2} \cos \overline{\phi_{s}} \psi=\mathcal{F}\left(\frac{d \psi}{d \tau}, \psi, \tau\right)
$$

where

$$
\begin{aligned}
& \mathcal{F}\left(\frac{d \psi}{d \tau}, \psi, \tau\right)=-\Omega_{0}^{2} \sin \overline{\phi_{s}} \frac{d \psi}{d \tau}-\Omega_{0}^{2} \cos \overline{\phi_{s}} \frac{\delta E}{E} \sum_{k} e_{k} \cos k \nu \tau \psi+\frac{1}{2} \Omega_{0}^{2} \sin \overline{\phi_{s}} \\
& \left(1+\frac{\delta E}{E} \sum_{k} e_{k} \cos k \nu \tau\right) \psi^{2}+\frac{\delta E}{E} \Omega_{0}^{2} \sin \overline{\phi_{s}} \sum_{k} e_{k} \cos k \nu \tau+2 \nu_{\beta} \delta \beta \sum_{n} \cos 2 \pi \nu_{\beta} n \tau .
\end{aligned}
$$

The first term of the right side describes the damping, the second term describes the linear parametric excitation, the third one inputs the nonlinear feature in parametric and external resonances, the fourth term is the external force, and the last one is oscillation of the average synchronous phase.

It is obvious to find the solution in form:

$$
\begin{aligned}
\psi & =a \cos \theta \\
\theta & =w \tau+\tilde{\theta} \\
w & =\Omega_{0} \cos ^{1 / 2} \overline{\phi_{s}} .
\end{aligned}
$$

The first approach of this equation can be for the case when the right side equals zero:

$$
\begin{aligned}
\psi & =a \cos \theta \\
\frac{d \psi}{d \tau} & =-a w \sin \theta .
\end{aligned}
$$

Then for an unhomogeneous equation we have:

$$
\begin{aligned}
& \frac{d \psi}{d \tau}=\frac{d a}{d \tau} \cos \theta-a \frac{d \tilde{\theta}}{d \tau} \sin \theta-a w \sin \theta \\
& \frac{d \psi}{d \tau}=-a w \sin \theta
\end{aligned}
$$

From here one follows

$$
\frac{d a}{d \tau} \cos \theta-a \frac{d \bar{\theta}}{d \tau} \sin \theta=0
$$




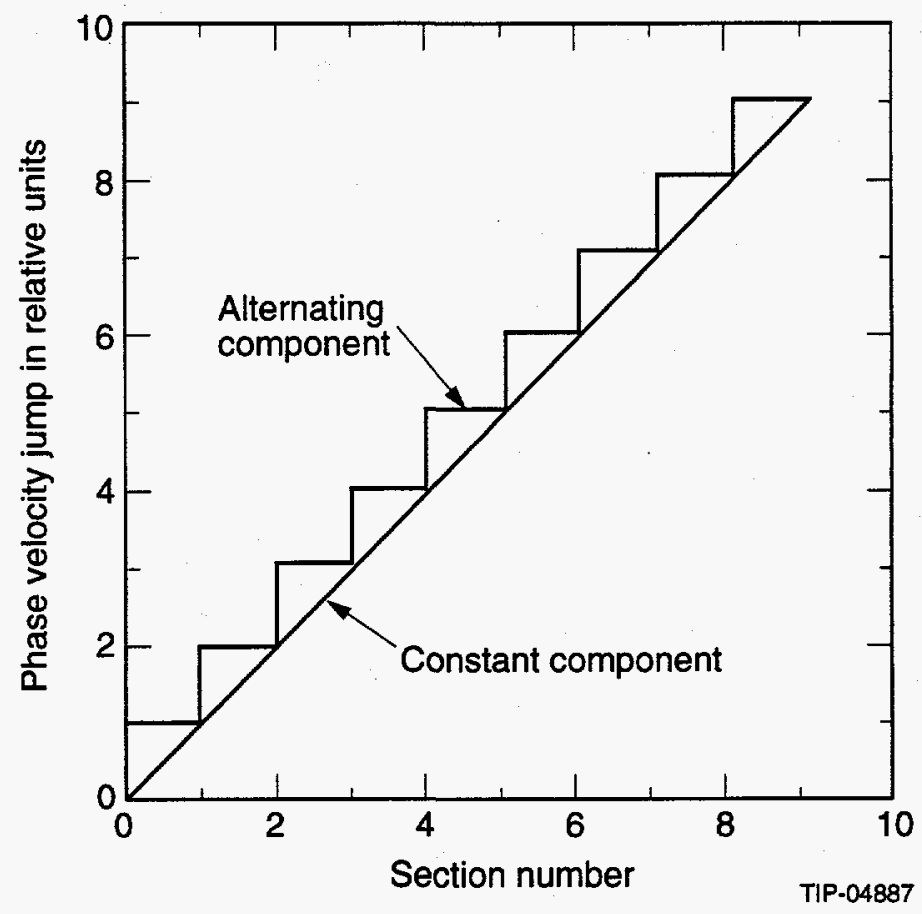

Figure 6 . The phase velocity vs. the section number.

Differentiating the second equation of system (55), we have:

$$
\frac{d^{2} \psi}{d \tau^{2}}=-\frac{d a}{d \tau} w \sin \theta-a w \frac{d \bar{\theta}}{d \tau} \cos \theta-a w^{2} \cos \theta
$$

Substituting both of these expressions in the initial equation and averaging over phase, we have:

$$
\begin{aligned}
& \frac{d a}{d \tau}=-\frac{1}{2 \pi w} \int_{0}^{2 \pi} \mathcal{F}\left(\psi, \frac{d \psi}{d \tau}, \tau\right) \sin \theta d \theta \\
& \frac{d \theta}{d \tau}=w-\frac{1}{2 \pi a w} \int_{0}^{2 \pi} \mathcal{F}\left(\psi, \frac{d \psi}{d \tau}, \tau\right) \cos \theta d \theta .
\end{aligned}
$$

Let's substitute the right side of Eq. (52) in these equations in order to get the first approach of the solution to Eq. (49). Since we are interested in the condition, under which the instability arises, it is possible to formulate so the threshold of instability originates when the derivative $\frac{d a}{d \tau}$ in average becomes positive.

The first term, which describes the damping, gives contribution in $d a / d \tau$ :

$$
\begin{aligned}
\frac{d a_{1}}{d \tau} & =-\frac{1}{2 \pi \Omega_{0} \cos ^{1 / 2} \overline{\phi_{s}}} \int_{0}^{2 \pi} \Omega_{0}^{2} \sin \overline{\phi_{s}} a \Omega_{0} \cos ^{1 / 2} \overline{\phi_{s}} \sin ^{2} \theta d \theta \\
& =-\frac{\Omega_{0}^{2} \sin \overline{\phi_{s}}}{2} a .
\end{aligned}
$$


It is obvious that the coefficient responsible for the damping is proportional to $\sim e^{-\frac{1}{2} \Omega_{0}^{2} \sin \overline{\phi_{s} \tau}}$, or $-\frac{1}{2} \Omega_{0}^{2} \sin \overline{\phi_{s}} \tau$, since $\Omega_{0}^{2}$ is a small value.

The second term, which is responsible for the parametric resonance gives the contribution in $d a / d \tau$ :

$$
\begin{aligned}
\frac{d a_{2}}{d \tau} & =\frac{1}{2 \pi \Omega_{0} \cos ^{1 / 2} \overline{\phi_{s}}} \int_{0}^{2 \pi} E_{k} \Omega_{0}^{2} \cos \overline{\phi_{s}} \cos k \nu \tau \cos \theta \sin \theta d \theta \\
& =\frac{\Omega_{0} \cos ^{1 / 2} \overline{\phi_{s}}}{4} E_{k} \sin (2 \theta-k \nu \tau+\bar{\theta}),
\end{aligned}
$$

where $E_{k}=\frac{\delta E}{E} e_{k}$ is the relative harmonic of perturbation and $\bar{\theta}$ is slow phase. In the case of the parametric resonance $\nu=\frac{\Omega_{0} \cos ^{1 / 2} \overline{\phi_{s}}}{k}$, maximum growth of amplitude will be $\Omega_{0} \cos ^{1 / 2} \overline{\phi_{s}} E_{k} / 4$. Consequently the threshold of resonance will equal:

$$
E_{k}=\frac{2 \Omega_{0} \sin \overline{\phi_{s}}}{\cos ^{1 / 2} \overline{\phi_{s}}}
$$

The fourth term, which is responsible for the external resonance, has value:

$$
\begin{aligned}
\frac{d a_{4}}{d \tau} & =\frac{1}{2 \pi \Omega_{0} \cos ^{1 / 2} \overline{\phi_{s}}} \int_{0}^{2 \pi} E_{k} \Omega_{0}^{2} \sin \overline{\phi_{s}} \cos k \nu \tau \cos \theta \sin \theta d \theta \\
& =\frac{\Omega_{0} \sin \overline{\phi_{s}}}{2 \cos \overline{\phi_{s}}} E_{k} \sin (\theta-k \nu \tau+\bar{\theta})
\end{aligned}
$$

The resonance arises at $k \nu=\Omega_{0} \cos ^{1 / 2} \overline{\phi_{s}}$ and the threshold will be:

$$
E_{k}=\Omega_{0} \cos ^{1 / 2} \overline{\phi_{s}}
$$

The external resonance has a threshold lower and it is more significant. In practice, for instance, this threshold has order $\sim 1 \div 2 \%$. The third term gives the nonlinear stabilization, which raises slightly the threshold for both resonances. The last term causes the coherent motion of all particles in the separatrix that is equivalent to the oscillation of the instantaneous synchronous phase. Figure 7 shows the synchronous frequency as a function of the cell number. 


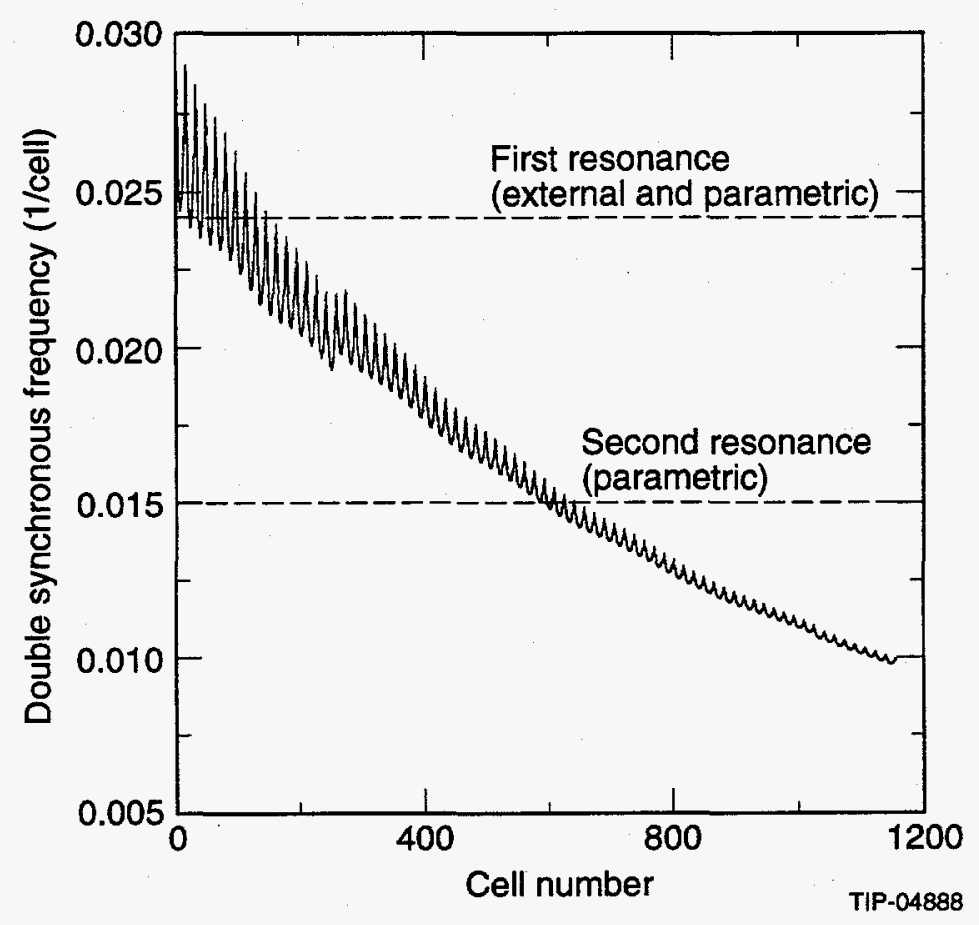

Figure 7. The synchronous frequency as a function of the cell number.

\subsection{NUMERICAL RESULTS AND DISCUSSION}

As an example we consider the Coupled Cavity Linac of the SSC. Figure 7 shows behavior of the quasi-synchronous frequency along the CCL accelerator. The vertical lines show the possible resonance frequency. Oscillation of the frequency is because the constant phase velocity of the tank. It increases the effective time of passing through resonance. Figures $8-$ 13 show the numerical simulation of the resonance passing particles. Each illustration shows "a" and " $\mathrm{b}$ " options for comparison. The option "a" is for the case of the ideal accelerator without perturbation and " $\mathrm{b}$ " is with perturbation. At the entrance to the accelerator we took "beam" with the distribution as five lines, each of which has a different velocity. That initial distribution is convenient for the resonance observation. Particles are connected each with other by lines. This representation has a small disadvantage. During the resonance the extreme particles are stretched so strongly that we lose some information. Otherwise this method gives a good representation of what happens in resonance.

In an ideal accelerator the action of fundamental resonance in longitudinal motion, which gives the acceleration, creates S-shaped lines. But already at Module 1 in Tank 6 we begin to feel the influence of external resonance. Since the frequency of perturbation is very near to the frequency of fundamental resonance, we observe slightly stronger modulation of the S-shaped figure at first. But then this modulation changes shape (see Figure 10). The creation of the tails is explained by the degeneration of the fundamental and external 
resonances. After some turns we can observe many tails. We can see in Figure 11 two tails and three tails in Figure 12. Figure 13 demonstrates the junction of all tails in the losses tail. It means passing many times through resonance with simultaneous sliding of frequency. The question is what to do? It is obvious we should go from the resonance. In other words, if the reason for the perturbation is the power input, let's change the wavelength of this perturbation. One solution is to input power at two symmetrical points at $1 / 4$ and $3 / 4$ of the module length. Then the amplitude of the wave will decrease two times and the frequency of the perturbation will increase two times giving the possibility of avoiding the resonant condition. Figure 12(c) shows the phase portrait of beam in the same module and tank when we input the power at two points. It is obvious that the picture becomes more similar to the ideal case (see Figure 12a).
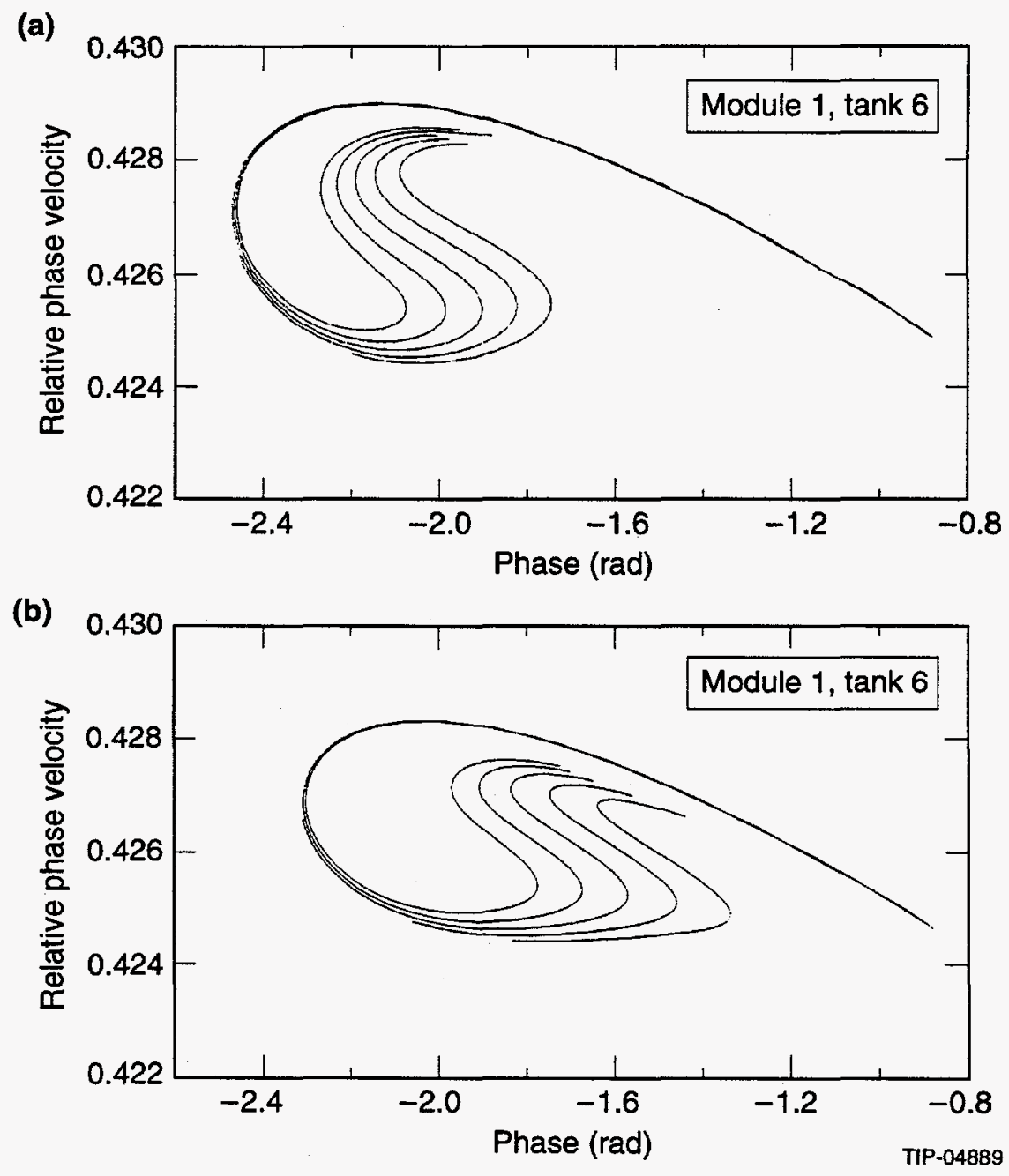

Figure 8. In the SSC CCL Module 1, Tank 6: (a) phase portrait for ideal field and (b) phase portrait for perturbed field. 
(a)

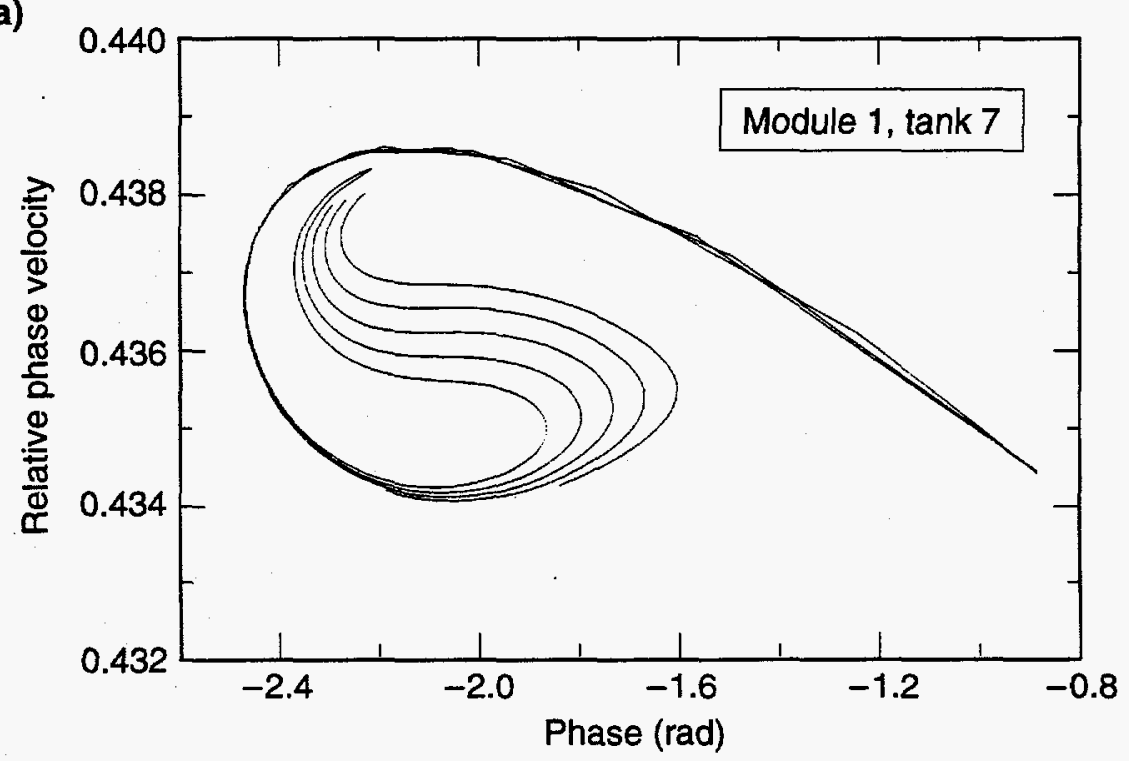

(b)

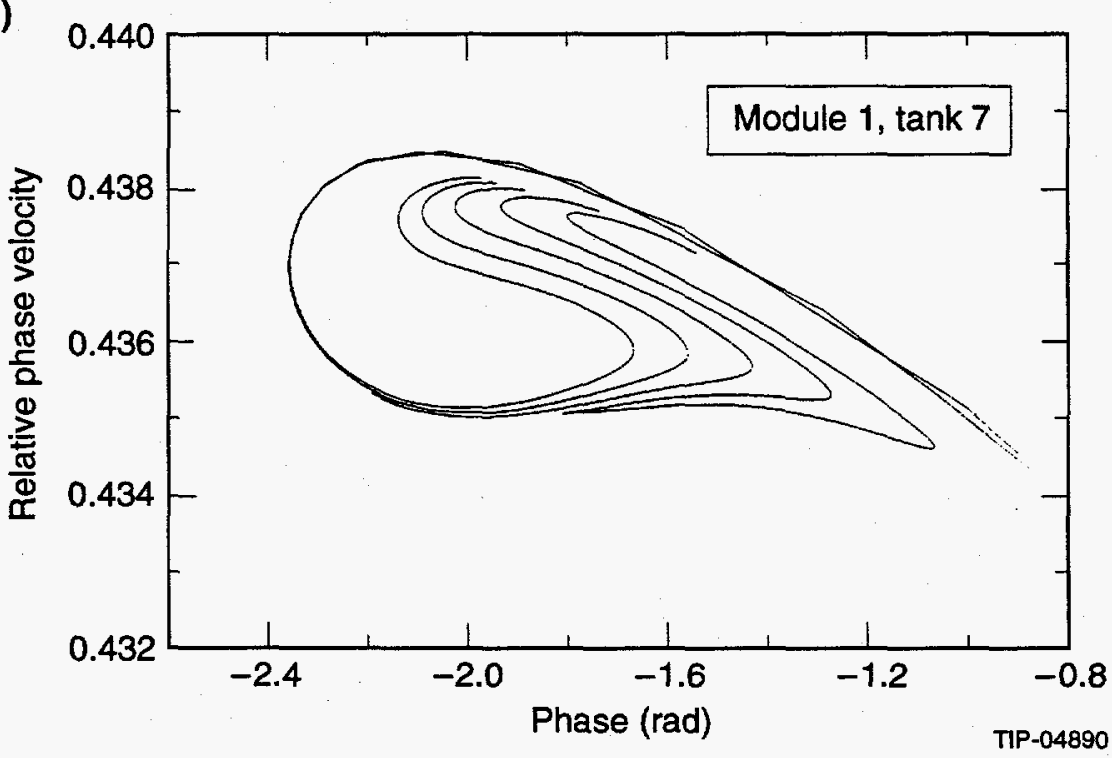

Figure 9. Module 1, Tank 7: (a) phase portrait for ideal field and (b) perturbed field. 
(a)

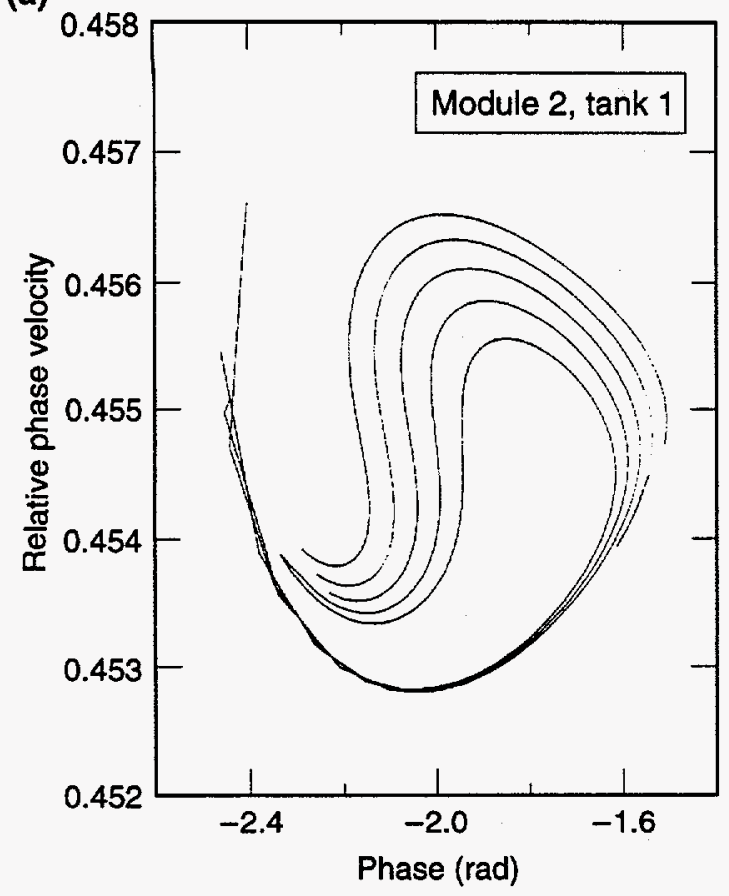

(b)

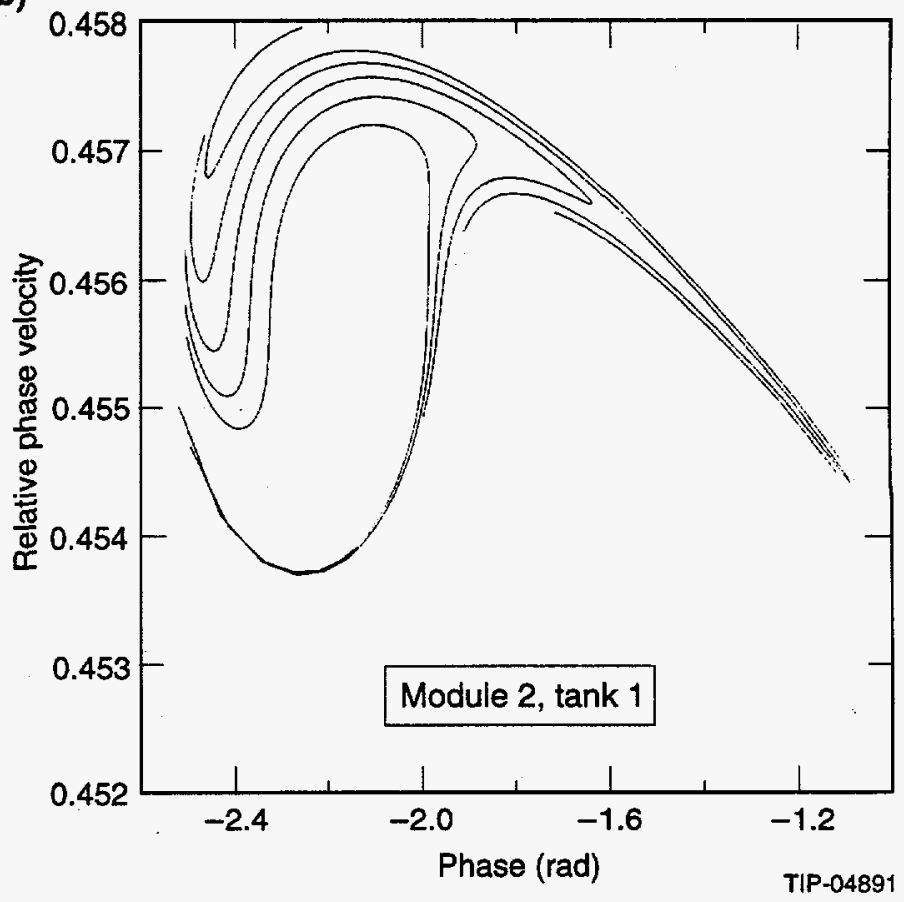

Figure 10. Module 2, Tank 1: (a) phase portrait for ideal field and (b) perturbed field.

(a)

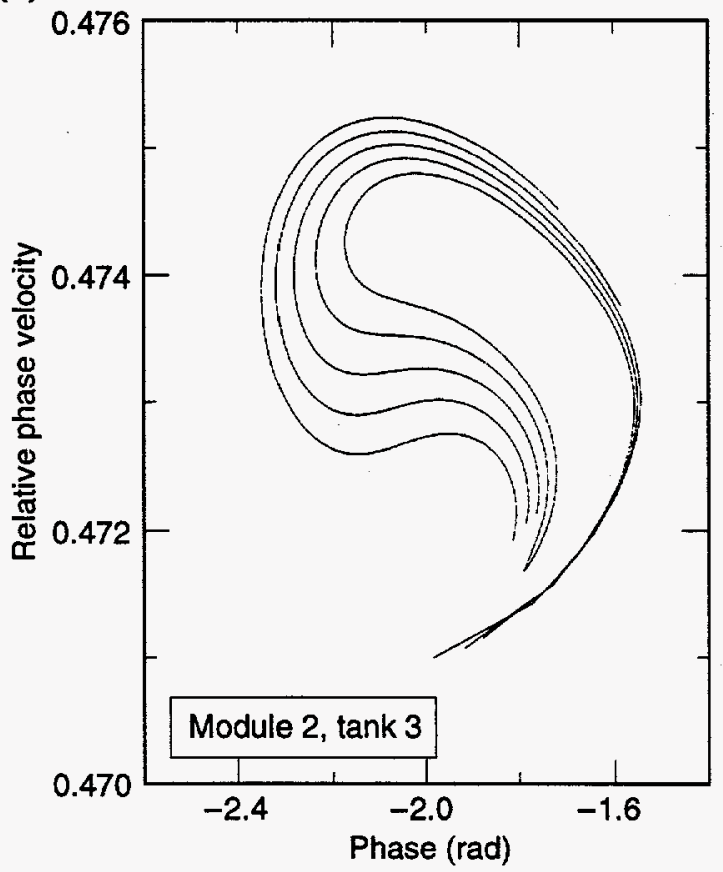

(b)

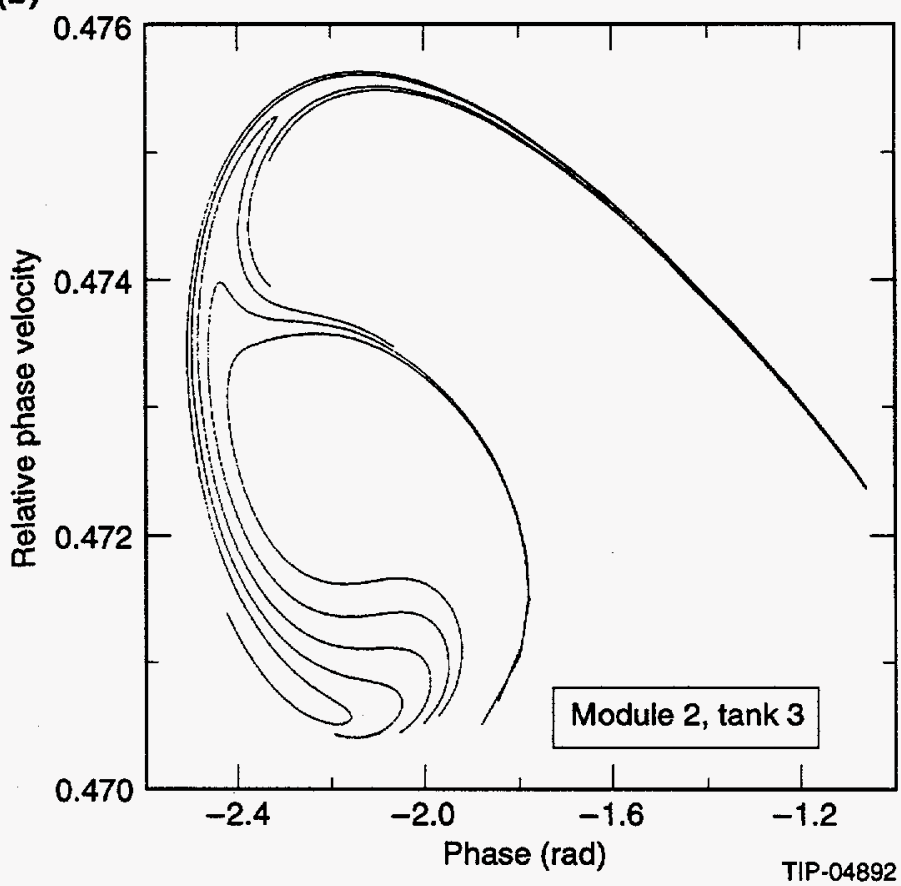

Figure 11. Module 2, Tank 3: (a) phase portrait for ideal field and (b) perturbed field. 
(a)

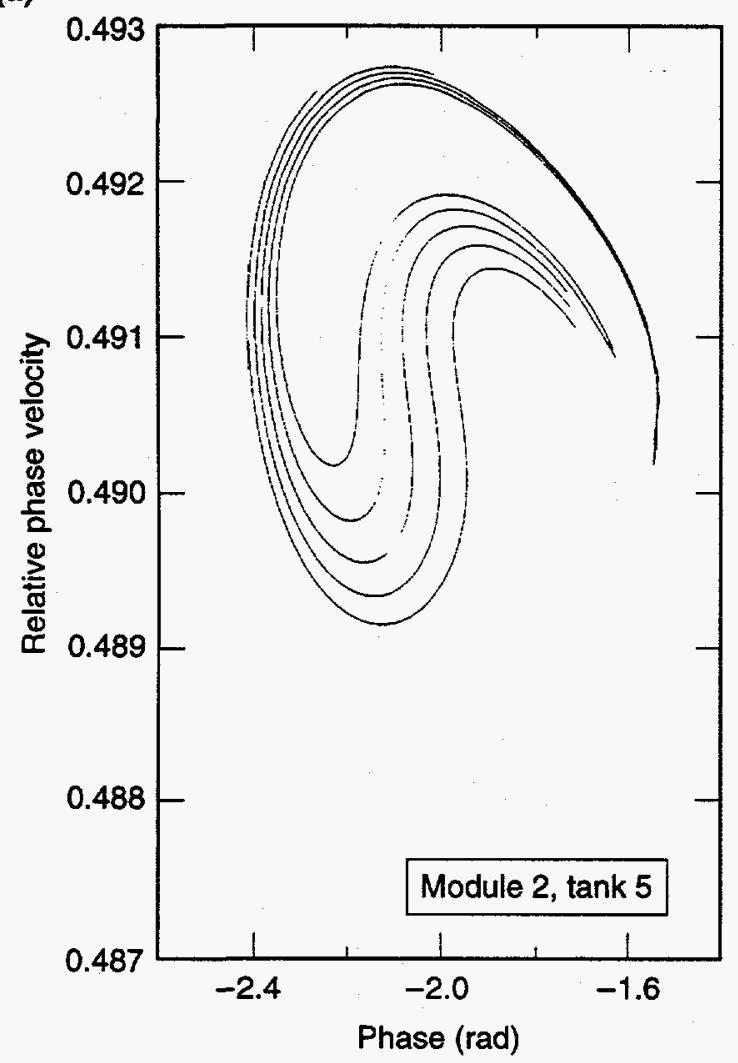

(c)

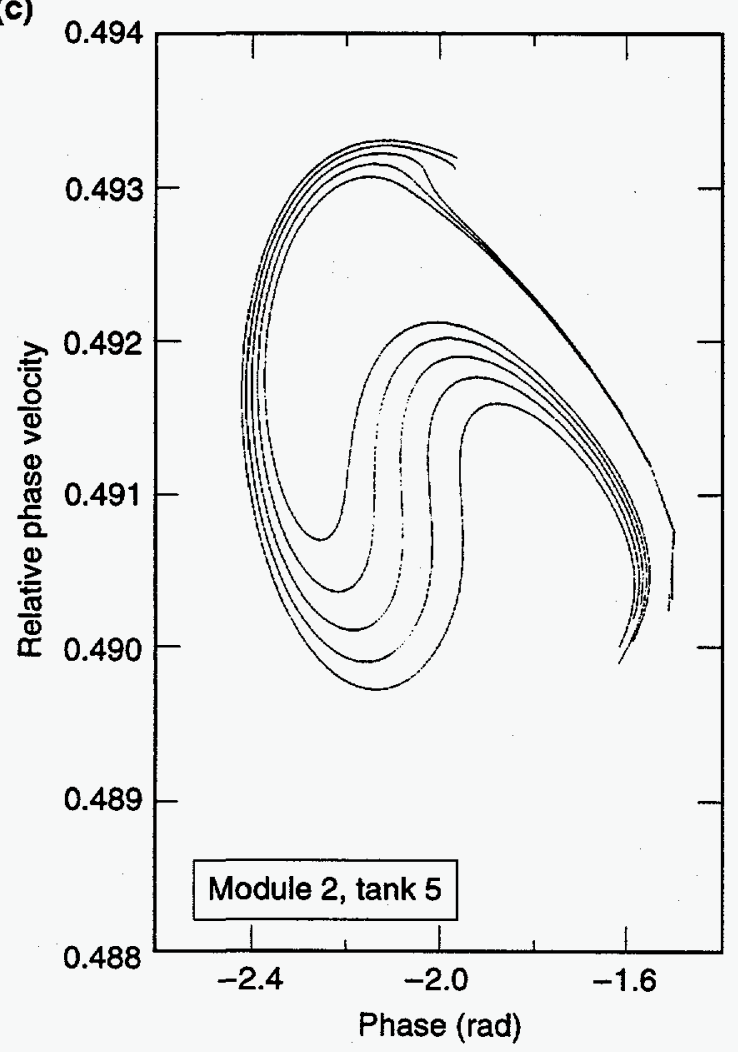

(b)

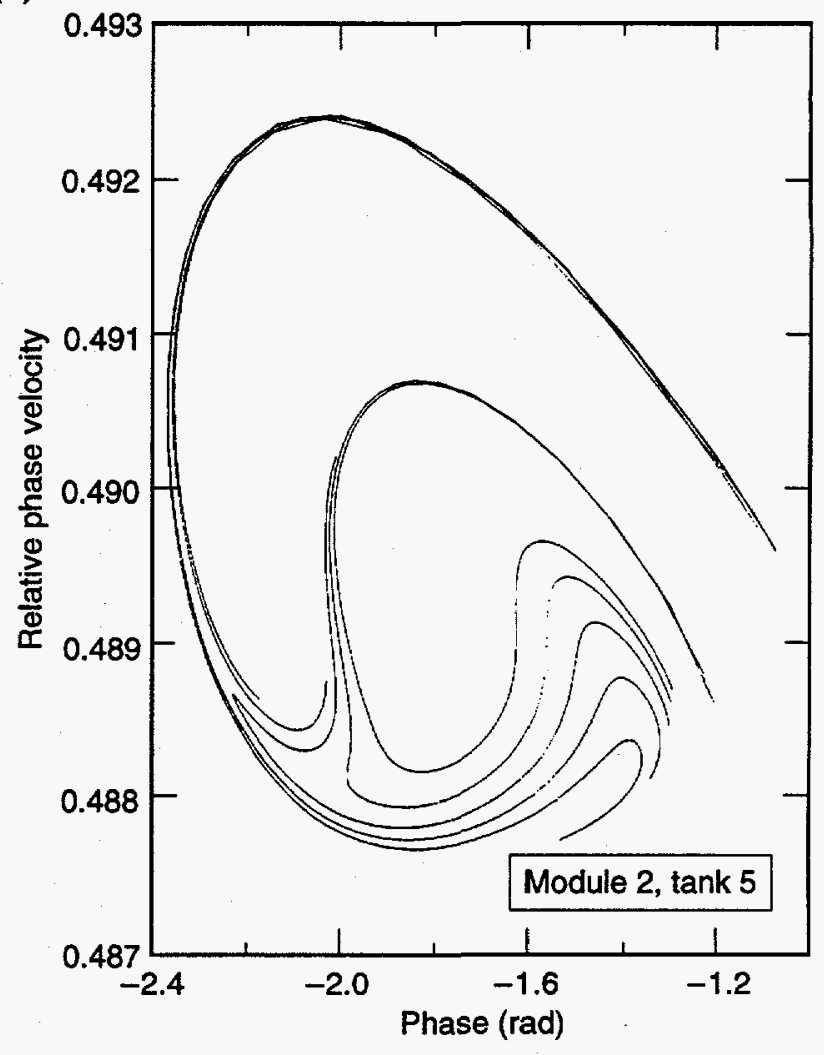


(a)

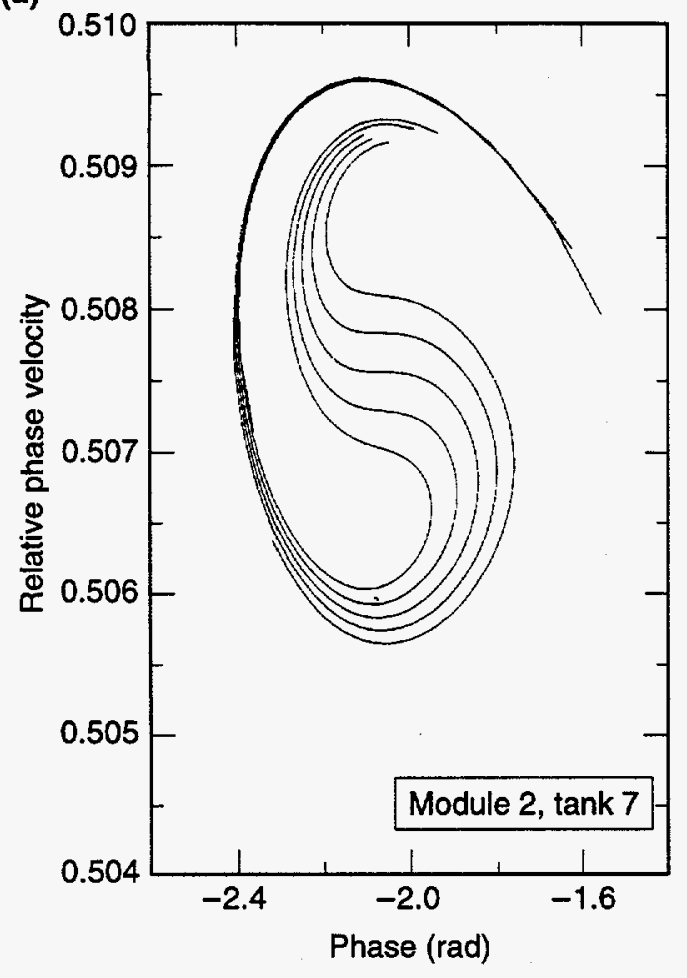

(b)

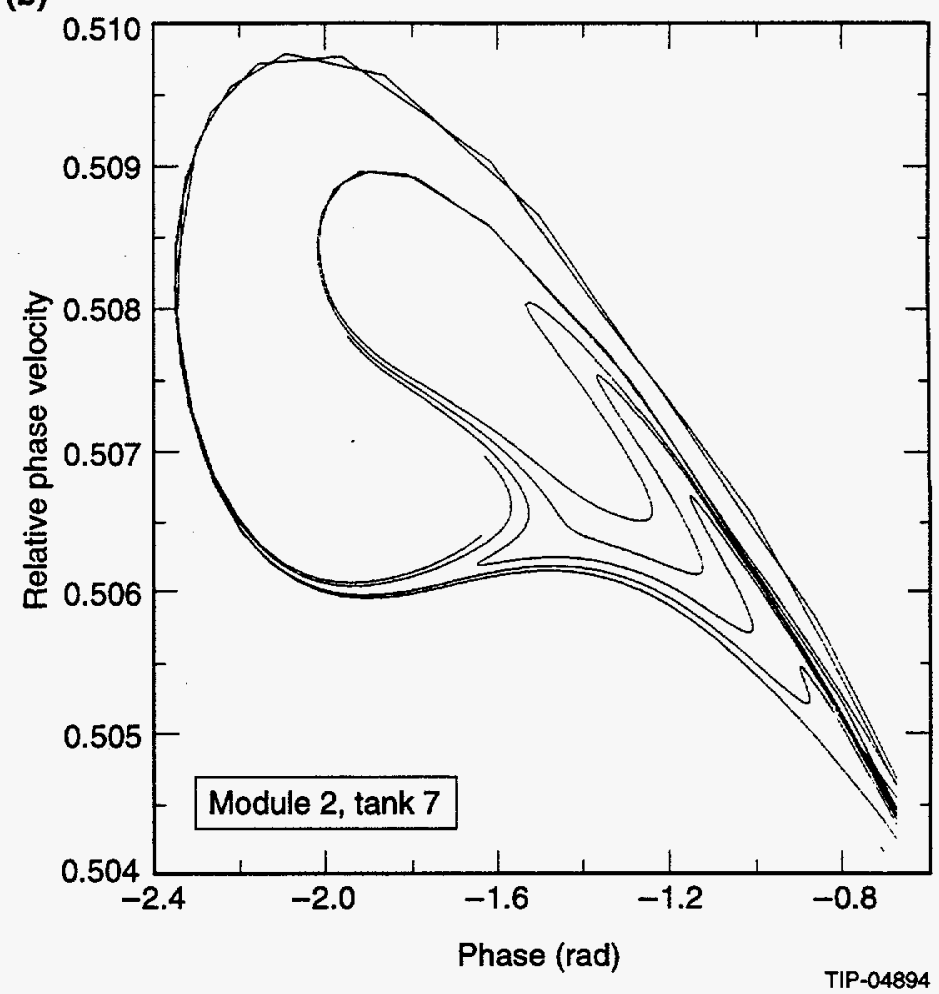

Figure 13. Module 2, Tank 7: (a) phase portrait for ideal field and (b) perturbed field.

\subsection{CONCLUSION}

In conclusion I would like to discuss recommendations for accelerator design, taking into account the transient effect studied in this paper. In any case, we have to accept the integer resonance passing since it gives the most restriction for the perturbation and consequently for the control system. The exclusion condition of the resonance passing can be written as:

$$
\Delta W_{\lambda} N^{2}<4 \pi \mathcal{E}_{0} \gamma^{3} \beta^{2} \tan \phi_{s},
$$

where $\Delta W_{\lambda}$ is the energy gain per one accelerating cell, $N$ is the number of cells in the resonator and $\mathcal{E}_{0}$ is the rest mass of a proton. One can see from here that the most effective method to avoid the passing resonance is the changing of the number of cells in the resonator. If the number of cells is fixed due to some reason (for instance due to radial periodicity), then the maximum acceleration rate is defined by the requirements for the low energy resonators:

$$
\Delta W_{\lambda}<\frac{4 \pi \mathcal{E}_{0} \gamma_{0}^{3} \beta_{0}^{2} \tan \phi_{s}}{N^{2}}
$$


where the index " 0 " means the initial parameters of the beam. If the generator power is fixed, that is $\Delta W_{\lambda} N=$ const, then $N$ has to be less than:

$$
N<\frac{4 \pi \mathcal{E}_{0} \gamma_{0}^{3} \beta_{0}^{2} \tan \phi_{s}}{\Delta W}
$$

where $\Delta W$ is the energy gain per one resonator. Since all requirements are defined at low energy, the most effective method is to use the multi-point input of power in the first resonators. 



\section{ACKNOWLEDGEMENTS}

I would like to express my thanks to Dr. W. Funk and Dr. J. Hurd for their interest and support. I thank to Dr. D. Raparia for helpful discussions during work, with whom author continues to study this effect on SSCL, LAMPH and FNAL. I thank also Dr. Chong Guo Yao and Dr. Chu Rui Chang for useful discussions and experimental measurements of modes. 


\section{REFERENCES}

1. R.A. Jameson, "Beam Halo from Collective Core/Single-particle Interactions," Los Alamos, NM 87545, LA-UR-93-1209, (1993).

2. Yu. Senichev et al., "Problems of the Beam Loss in Intensive Ion Linear Accelerators," 1988 Linear Accelerator Conference Proceeding, CEBAF-Report-89-001.

3. Yu. Senichev, "Study of Injection Transient in Coupled Cell Linac Cavities," Third European Particle Accelerator Conference, pp.774-776, Berlin, (1992).

4. G. Zaslavsky, Stochastic Dynamic System, Moscow (1984).

5. B. Chirikov, Nonlinear Resonance, Novosibirsk (1977).

6. N. Bogoljubov, Asymptotic Method in Nonlinear Oscillations Theory, Moscow (1958). 